\title{
¿Educación moral para la autonomía? Fundamentos y contradicciones
}

\author{
Moral education for autonomy? Basis and contradictions
}

\author{
Betzabeth Reyes Pérez \\ Universidad Nacional Autónoma de México \\ bethrepe@gmail.com
}

\begin{abstract}
Resumen
A partir de la ilustración se presumió de la libertad de autogobernarse, el ser humano pasó a ser el centro de lo existente y por lo tanto, ser responsable de lo que sucedía. Kant es quien dio un giro copernicano al estudio moral, incluye como una de sus más importantes aportaciones morales el estudio de la autonomía, que constituye la tercera fórmula del imperativo categórico. A mi consideración éste podría ser el fundamento filosófico de la educación moral, pues posterior a Kant Piaget se encargó de realizar estudios sobre la moral en los niños. Tarea que complementó Kolhberg y que desencadeno en la propuesta de educación moral que permeó los programas de educación en México. Formar sujetos autónomos es una de las ambiciones que la mayoría de los sistemas educativos pretende, incluyendo el nuestro. Parece ser un interés cada vez más ambicioso, particularmente explicitado en los programas de Formación cívica y Ética en educación secundaria. Conocemos las condiciones desfavorables que hacen convulsionar nuestro país y ante esas problemáticas, se le ha dado a la escuela la responsabilidad para evitar (en alguna medida), escenarios decadentes de vida a través de educación moral por medio de la asignatura antes referida en educación secundaria. El análisis de los elementos provistos en esta asignatura se inclina en gran medida hacia una conceptualización de autonomía desde el punto de vista kantiano. Tomo elementos de ésta concepción para discutir posteriormente si es o no posible la enseñanza de la misma.
\end{abstract}

\section{Palabras clave \\ Autonomía, educación, moral, imperativo, ética.}

\begin{abstract}
From the illustration period, the freedom to self-govern was presumed, the human being became the center of what exists and, therefore, be responsible for what happened. Kant is who gave a Copernican twist to moral study, including as one of his most important moral contributions the study of autonomy, which constitutes the third formula of the categorical imperative. To my consideration, this could be the philosophical foundation of moral education, because, after Kant, Piaget conducted studies regarding morality in children. This task was complemented by Kolhberg and unleashed the moral education proposal that permeated the education programs in Mexico. To form autonomous subjects is one of the ambitions intended by most educational systems, including ours. It seems to be an increasingly ambitious interest, particularly explicit in the programs of civic education and ethics in middle school. We know the unfavorable conditions that make our country convulse and in front of these problems, the school has been given the responsibility to avoid (to
\end{abstract}


some extent), decadent life scenarios through moral education using the previously mentioned subject in middle schools. The analysis of the elements provided in this subject lean largely towards a conceptualization of autonomy from the Kantian point of view. I take elements of this conception to discuss later whether it is possible to teach it.

\section{Keywords}

Autonomy, education, moral, imperative, ethics.

\section{Problemática}

La propuesta de enseñanza de la autonomía en educación secundaria es uno de los ejes fundamentales en la asignatura de Formación Cívica y Ética, sin embargo el control y la coacción impera en este nivel educativo. En ese sentido se vive una tensión entre dos disciplinas: la pedagogía a través de su establecimiento curricular y la filosófica a partir de las elaboraciones éticas respecto a lo moral y en particular a la autonomía. Al hacer frente a las dos disciplinas, requerimos conciliar y comparar elementos de análisis para una elaboración más profunda de los programas que tienen que ver con la educación moral. Esto es porque en muchas etapas históricas esta formación ha tratado de ser desaparecida, sin reconocer la importancia de la misma, pero al mismo tiempo sin darle la seriedad requerida en la construcción de los contenidos que se quieren impartir. Por esta razón considero necesario un análisis pedagógico y filosófico de la concepción de autonomía que se pretende enseñar a través de los documentos oficiales de la Secretaría de Educación Pública.

\section{Preguntas}

¿Qué cuño es la concepción de autonomía de los programas de Formación Cívica y Ética de educación secundaria?

¿Qué hacen los programas de Formación Cívica y Ética de educación secundaria en favor de la autonomía?

¿De qué manera sería posible la enseñanza de la autonomía como un acto moral?

\section{Objetivos}

Conocer el cuño del concepto de autonomía con el que se elaboraron los programas de estudio de la materia de Formación Cívica y Ética en educación secundaria.

Elaborar un análisis comparativo con bases pedagógicas y filosóficas entre el texto curricular y el referente filosófico encontrado.

Problematizar la enseñanza de la autonomía como tarea de la educación moral dentro de la escuela a partir de los programas de estudio de la asignatura antes señalada.

\section{Metodología}

La investigación presentada se posiciona en el desarrollo de la Filosofía de la Educación. Para esto acuño como metodología la Hermenéutica Analógica, que es caracterizada por considerar diversos elementos en discusión y posiciones distintas para buscar una conciliación que fundamente la interrelación de elementos compartidos. No anula alguno de ellos, ni nace como respuesta a uno de los extremos para fundarse como verdadero método de interpretación, sino que a partir de la concepción histórica de las diversas perspectivas y de los elementos que las conforman, se construye una propuesta que permite ver un equilibrio. Esto es una interpretación analógica o limítrofe, cuando se intenta conciliar pero no equiparar los diferentes textos (Beuchot, 2002).

Así la Hermenéutica Analógica es una herramienta de interpretación que me ayuda a situar los dos ejes principales de mi tesis, a saber: la propuesta de autonomía Kantiana 
elaborada en el siglo XVIII, y por otro lado la propuesta educativa expresada en los programas de Educación Secundaria de 2011. El tiempo recorrido entre ambos escritos no es menor, al considerar casi tres siglos de diferencia, algunos podrían notar desfases e imposibilidad del análisis a causa de lo histórico y espacial. Sin embargo, el uso de la interpretación analógica como metodología ayudará a retomar elementos necesarios de ambos textos para la realización de una interpretación entre ellos.

Beuchot establece que "los textos son de varias clases: pueden ser escritos, hablados e incluso actuados. Todo lo que tiene una significación viva, no completamente inmediata y clara, es susceptible de interpretación"(Beuchot, 2002:86). Considero pues, este modelo necesario para la interpretación de la educación, así como de su fundamentación filosófica como un texto en tanto que se materializa en interacción educativa.

El siguiente trabajo tiene como objetivo describir el avance de resultados del trabajo de investigación teórica que realizo. A partir de la ilustración se presumió de la libertad de autogobernarse, el ser humano pasó a ser el centro de lo existente y por lo tanto, ser responsable de lo que sucedía, ya no lo era una deidad o fuerza sobrenatural quien tenía el control de los hechos, sino él mismo. Kant es quien dio un giro copernicano al estudio moral dentro del idealismo, incluye como una de sus más importantes aportaciones morales el estudio de la autonomía. Ésta constituye la tercera fórmula del imperativo categórico, que a mi consideración podría ser el fundamento filosófico de la educación moral, que se considera vigente y que trastoca al ser humano como fin, capaz de deliberar, a partir de su racionalidad.
Formar sujetos autónomos es una de las ambiciones que la mayoría de los sistemas educativos pretende, incluyendo el nuestro. Formarlos con una característica que afecte su moralidad, parece ser un interés más ambicioso cuando conocemos las condiciones desfavorables que hacen convulsionar nuestro país. El proyecto de investigación que trabajo, parte de conceptualizaciones filosóficas que explican la fundamentación de los programas de estudio de la materia de Formación Cívica y Ética 1 y 2 de Educación Secundaria. Mi interés particular se encuentra en el sentido del uso del concepto de autonomía que se procura.

En primer lugar, se encuentra en uno de los objetivos principales y reza de la siguiente manera: [que los estudiantes] "Comprendan que los derechos humanos y la democracia son el marco de referencia para tomar decisiones autónomas que enriquezcan la convivencia, el cuestionar acciones que violen el derecho de las personas y afecten su ambiente natural y social”. (SEP, 2011).

En el objetivo anterior se comprende a la autonomía como el fin establecido a partir del uso de ciertos medios (derechos humanos y democracia) para alcanzarlo, a lo largo del programa podemos ver que la dirección se encuentra en esta finalidad. Un ejemplo más se localiza en el segundo apartado de las "Competencias cívicas" que el programa sugiere. Ésta corresponde a la siguiente premisa: "Autorregulación y ejercicio responsable de la libertad", es necesario resaltar que la autonomía es concebido como sinónimo de la libertad. Éstos son sólo un par de ejemplos para demostrar que la autonomía juega un papel importante en el desarrollo del programa de la asignatura antes mencionada.

Por esa razón el concepto de "Autonomía" es el eje medular de mi investigación, pues a través de un rastreo filosófico encuentro que el primer pensador en usar este concepto es Emanuel Kant, de ahí 
la relevancia de recurrir a sus postulados filosóficos. De esta manera he decido dedicar énfasis al planteamiento de la problemática existente entre la relación del uso del concepto en los programas de estudio y su implicación estrictamente filosófica.

La investigación enmarca una visión filosófica que sustenta la propuesta moral kantiana, que precede a la concepción de autonomía que se encuentra en los programas de estudio de educación secundaria. La construcción de este primer marco filosófico, involucra otras categorías en torno a la ética idealista y la propuesta kantiana que se requiere comprender para buscar el cometido establecido en los programas de estudio. Sin embargo la definición de categorías no se limita solamente a una propuesta moral, sino a su implicación histórica y educativa que prevalece hasta hoy.

La autonomía de la voluntad definida por Kant "es la constitución de la voluntad, por la cual es ella para sí misma una ley" (Kant, 2013:57), ésta es el fundamento del imperativo, en tanto que se gobierna así misma, es decir: se autolegisla, elige máximas que también pueden ser leyes universales, se somete a la ley de la que ella misma es autora, es decir, considera una máxima como imperativo que puede ser categórico. La autonomía es el único principio de la moral, donde ésta última establece la relación de las acciones con la autonomía de la voluntad. La voluntad autónoma y la voluntad moralmente buena son la misma cosa, y es buena, siempre y cuando actúe según leyes universales, es decir, que actúe con el imperativo categórico.

La voluntad autónoma tiene un carácter incondicionado del imperativo porque supone que la voluntad no se somete a él por la mediación de ningún interés, es decir, “[...] la idea de una legislación universal no se funda en interés alguno y es, de todos los imperativos posibles, el único que puede ser incondicionado" (Kant, 2013:51), puesto que si se fundará en un interés sería restringida por un elemento externo a ella misma, y en este caso requeriría otra ley para regularse.

La voluntad no funciona a partir de condicionantes que determine el objeto, es decir, no se limita por él, no depende de él, y el interés es para Kant "la dependencia de una voluntad determinable contingentemente respecto de los principios de la razón" (Kant Cit. Rivera, 2014:167); por lo tanto, si la voluntad depende del interés ya no se expresa como autónoma, pues puede ser determinada por otro agente, Kant afirma que la relación entre la voluntad y los principios de la razón no pueden estar mediados por los intereses, si eso sucede, no podemos hablar de un imperativo categórico.

Por el contrario, la voluntad moralmente buena (autónoma), se fundamenta en el imperativo categórico y queda según Kant "indeterminada respecto de los objetos y contendrá sólo la forma del querer en general, como autonomía [...] sin que intervenga como fundamento ningún impulso e interés" (Kant, 2013:61), lo que se da es la forma, el contenido no está determinado, a partir de la elección de máximas se podrá verificar si son posibles sus universalizaciones, entonces podremos decir si es o no un imperativo categórico, que como ya se dijo antes, no es para juzgar los actos ajenos, sino para evaluación de los propios.

Ahora la pregunta es ¿cómo lograremos un sujeto autónomo? Frente a esta problemática es necesario echar mano del acto educativo, Kant afirma que "el hombre es la única criatura que ha de ser educada" (Kant, 2003:29), sus condiciones racionales le permiten transformarse. Trabajar una educación moral para la autonomía, pretender un sujeto que sea capaz de decidir racionalmente, considerado al otro y así mismo como un fin y no como un medio, es romper con una educación moral hasta hoy pretendida, que está paralizada, que esta 
utilitarizada para buscar éxito económico y profesional a costa del otro como medio.

Esta condición tiene como consecuencia un conformismo moral, que parte de modelos supremos de valoraciones como definitivas que no pueden transgredirse y que se señalan como fines últimos que logran la dogmatización del ser humano, pero que lo dirige su amor propio (su egotismo, diría Kant), y da como resultado la resistencia al cambio, el rechazo a lo nuevo, sin pensar que la moral está en constante crisis, puesto que no puede permanecer estática, en tanto que es propia del sujeto.

Romper con la educación moral que hasta hoy no ha funcionado, significa cambiar los modelos y considerar una educación para la autonomía, que como menciona Mougán (2013:2) es "aquel tipo de educación que permite considerar las cosas desde distintos ángulos, que se aleja de una consideración superficial o simplista de las cuestiones". Significa un cambio de rumbo, en el que el sujeto deje de culpar entidades externas, asuma su responsabilidad. Desde una mirada kantiana, podríamos decir que sea un sujeto responsable de sí, que tenga en mente una máxima que pueda concientizar y lograr universalizar.

Partimos del supuesto que en las relaciones de enseñanza, se da cierto contenido moral, no puede separarse la manera en que el docente enseña, de sus convicciones morales, sus juicios de valor, de sus valoraciones; $\mathrm{y}$ en ese sentido, la enseñanza en general, también puede considerarse un espacio para la educación moral. En los espacios de enseñanza como la escuela, no se ha de considerar a la moral como un fragmento o una parte de los currícula, que regularmente tiene una desarticulación con el demás contenido, sino que ha de considerarse incluido en los diferentes apartados, o sea, en las actividades que corresponden a las diferentes materias.
El ser humano nunca podrá eliminar de su persona el contenido moral, es propio de él, y por lo tanto está presente en cada actividad que realiza, actúa con ella en donde quiera que está, puede realizar actos morales en las diferentes materias de la escuela, pero es importante que éstas actividades sean dirigidas o redirigidas por el educador, con el fin de que el alumno sea consciente de su actuar moral, que el alumno pueda darse cuenta de la articulación que existe entre su acción y su rol en la escuela, sus participaciones en las clases, sus relaciones con sus compañeros o los profesores.

A través de la educación siempre se ha intentado mejorar la conducta humana, se piensa en un fin específico y se intenta marchar con la pretensión de lograr objetivos establecidos, no solamente en contenidos curriculares, también en la moral del educando. Actualmente en nuestro país se pretende una acción similar, pero se parte de un listado de valoraciones que ha realizado agentes externos de la escuela como cadenas televisivas y otros medios de comunicación han decidido como los adecuados, el problema es que este mismo tipo de programa se pretende anclar en educación básica, fundamentados en reconocimiento y memorización de conceptos, actividad de Kant critica fuertemente en la Doctrina del método (2011).

Se pretende enseñar moral a partir de significados de conceptos considerados como acciones bondadosas para el progreso de la sociedad, mientras que en el discurso, se busca un ser humano consciente $\mathrm{y}$ responsable de sus actos. La pretensión de esta enseñanza y el objetivo que se quiere lograr distan en función y contenido, debido a que no se nota un proceso de reflexión desde que vemos las planeaciones de estos programas, existe una incoherencia entre los objetivos fijados y las actividades emprendidas. 
La educación moral para la autonomía no se trata de discursos bien intencionados aunque vacíos, se necesita de una intervención real, en la que los sujetos sometan a reflexión lo que acontece en su alrededor, se trata de una coherencia entre el decir y el hacer, de poner la apuesta en la racionalidad del otro, y se expone claramente que de manera general, el sujeto se anula cuando reacciona en función de lo que se le pide y se niega la posibilidad de reflexión, se disminuye su dignidad, cuando se somete a lo que se ha dado por terminado y continúa con ese fin sin considerar sus posibilidades de decisión reflexionada.

Educación moral no se trata de reproducir modelos establecidos o considerados buenos, si eso se hace, se adoctrina a las personas. Para Salmerón "la educación moral no consiste tan solo en la adquisición de algunos hábitos y en el progreso de ciertos conocimientos; consiste además en el desarrollo de la capacidad para comprender las acciones humanas" (Salmerón, 2000:383). Es necesario reconsiderar el papel del educador, para que el estudiante pueda ser capaz de representar la ley y construir máximas que puedan universalizarse, habría que considerar las sugerencias que Kant hace al respecto, pues no se trata de legalizar una acción, es decir, establecerla como legitimada y que todos la reproduzcan en tanto que beneficia a lo demás, se trata de crear moralidad en las convicciones, que las acciones sean fundamentadas en la racionalidad y el deber, no dirigidas por lo considero bueno; la propuesta moral de Kant sobrepasa ese modelo filosófico estableciendo el deber.

Kant argumenta que la manera en que un educador puede colaborar en la educación moral del otro, es a través de la observancia de las máximas morales como único móvil para el bien, que la acción no se funde en una actitud buena o con buena intención, sino que se establezca a partir de la representación del deber. Para ello será necesario considerar dos sugerencias:

1. "Hacer que el juicio [según leyes morales] sea una ocupación natural que acompañe nuestras acciones libres como las de los demás." (Kant, 2011:184) Es decir, que la acción pueda considerarse objetivamente conforme a la ley moral, ley que creo el propio sujeto a partir de la universalización de máximas, debido a que es propia del deber. Este juicio puede ser ejercitado a través de ejemplos criticados y trasladados contextualmente, en los que los estudiantes puedan reflexionar lo que se ha vivido, y no solamente adoptarlo como actitudes buenas; evitar que se guíen por entusiasmos o tristezas, que tenga como consecuencia la creación de soñadores.

2. "Atraer la atención con la vívida exhibición de la convicción moral [...] fijar la atención del discípulo sobre la conciencia de su libertad" (Kant, 2011:188), intentar que reflexione sobre la perfección negativa de la voluntad, la influencia de las inclinaciones, la libertad y el deber. Lo anterior para lograr una conciencia sobre la dependencia en las inclinaciones, que los humanos podemos tener y que perjudican el fin mismo, a saber, al ser humano.

Los puntos anteriores, mencionados de manera sucinta y quizá hasta reduccionista, son parte del método que Kant, puede considerar como favorable para crear una conciencia de la voluntad en el sujeto, conciencia regulada por el imperativo categórica que pos cuestión sintética concluye en la autonomía moral del sujeto. Aunque la moral puede pensarse a priori, Kant aseverará la pertinencia de la reflexión práctica, debido 
a que la realidad del sujeto constantemente lo afecta, la razón pura práctica radica en el análisis moral que el sujeto hace, con base en el deber, pero no para juzgar la conducta moral del otro, sino para someterse a sí mismo a un examen moral que le permita ser consciente de su propia acción. Kant cierra el estudio de la Doctrina del método con la siguiente idea: "Con esto sólo he querido señalar las máximas más generales de la doctrina del método de una educación y de una práctica morales" (Kant, 2011:189).

En conclusión, la autonomía es el principio moral que dirige la voluntad a través del deber, con la formulación de máximas que puedan ser universalizables y que consideren al ser humano como fin en sí mismo, no como un medio, la educación moral para la autonomía no puede aprenderse a partir de la imitación, o de la comprensión de conceptos aislados, se realiza a través de un proceso de racionalización en el que el sujeto es capaz de concientizarse, a través de una estrecha vinculación entre la propia razón y los fenómenos que suceden, es capaz de concebirse en lo sensible y lo inteligible.

\section{Referencias}

Beuchot, M. (2002) Perfiles esenciales de la hermenéutica. Ciudad de México, Universidad Nacional Autónoma de México.

Cortina, A. y Martínez E. (1996) Ética. España: Akal.

Cullen, C. (2004) Autonomía moral, participación democrática y cuidado del otro. Buenos Aires: Noveduc.

Kant, I. (2013) Fundamentación de la Metafísica de las costumbres. México: Porrúa. (2011) Crítica de la razón práctica. México: FCE-UAM-UNAM. (2003) Pedagogía. México: Akal.

Korsgaard, C. (2011) La creación de reino de los fines. México: UNAM, UAM, UACH.

Mougán, J. (2013) “Autonomía” en Diccionario Iberoamericano de Filosofía de la Educación. Doc. inéd. México: UNAM.

Rivera, F. (2014) Virtud, felicidad y religión en la filosofía moral de Kant. México: UNAM.

Salmerón, F. (2000) Filosofía y Educación. México: El Colegio Nacional.

SEP. (2011) Programas de estudio 2011. Guía para el maestro. Educación básica secundaria. Formación cívica y ética. Ciudad de México: SEP. 
RECIE. Revista Electrónica Científica de Investigación Educativa Vol. 3, núm. 2, enero-diciembre 2017, pp. 847-853. 